\title{
THE AUTOMATED SYSTEM FOR PARAMETRIC AND FUNCTIONAL CONTROL OF THE LOGIC ICS
}

\author{
George Davydov, Anna Kolosova*, Adeliya Sharipova, Dmitriy Boychenko \\ National Research Nuclear University MEPhI (Moscow Engineering Physics Institute), 115409, \\ Moscow, Russia
}

\begin{abstract}
The article discusses implementation of the automated system based on the use of National Instruments PXI platform and designed for parametric and functional control of the logic ICs. The features of specialized software and typical results, obtained using the developed system, are observed.
\end{abstract}

\section{Introduction}

Logic ICs are widely used in all complex systems and devices. The main advantage of logic elements, in comparison with other digital chips, is their high performance (low delay times), as well as low supply power. During the certification tests it is often necessary to develop special software for each type of IC separately $[1,2]$. The universal automated system developing is an urgent task for unification of the radiation test for different types of logic ICs. The work is dedicated to the development of an automated system for parametric and functional control of the logic ICs.

\section{Equipment and software}

The automated system has been developed for this research is based on National Instruments PXI platform [3] and consists of chassis with modular measurement units, as well as specialized software.

The following hardware was used in the developed system: NI PXIe-1073 (chassis), NI PXI-4110 (power supply), NI-6229 (digital and analog data acquisition), NI-5114 (oscilloscope), NI-2529 (relay switches), NI-6723 (digital and analog output), PXIe-6556 (high-speed digital input/output with PPMU and 200MHz bandwidth).

The specialized software was designed using the NI LabVIEW for manage the modular devices.

\footnotetext{
* Corresponding author: askol@spels.ru
} 


\section{Solution}

The system is designed as the real-time monitor of the main parameters of ICs before, during and after irradiation. During the influence, the device under test (DUT) is operating in one of the preset electric modes (static / dynamic mode, low power mode, "shutdown" mode, etc) [4]. The most typical parameters of DUT are shown in Table 1.

Table 1. Typical parameters for logic ICs.

\begin{tabular}{|c|c|c|}
\hline \multirow{2}{*}{ Parameter } & \multicolumn{2}{|c|}{ Range } \\
\cline { 2 - 3 } & min & max \\
\hline Power Supply current $\mathrm{I}_{\mathrm{CC}}$ & $\leq 1 \mathrm{nA}$ & $\geq 100 \mathrm{~mA}$ \\
\hline Input current $\mathrm{I}_{\mathrm{I}}$ & $\leq 1 \mathrm{nA}$ & $\geq 100 \mathrm{~mA}$ \\
\hline High output voltage $\mathrm{V}_{\mathrm{OH}}$ & $-1 \mathrm{~V}$ & $\mathrm{Ucc}(0 \ldots 6 \mathrm{~V})$ \\
\hline Low output voltage $\mathrm{V}_{\mathrm{OL}}$ & $-1 \mathrm{~V}$ & $\mathrm{Ucc}(0 \ldots 6 \mathrm{~V})$ \\
\hline Functional control & Static & $\leq 100 \mathrm{MHz}$ \\
\hline
\end{tabular}

Any test means the different regimes of DUT for measurement of each its parameter. Modes and parameters are regulated in DUT's datasheet, which usually include typical and specific parameters of IC.

The system software allows saving measurement results in useful and friendly format.

Figure 1 illustrates the control panel of the developed system.

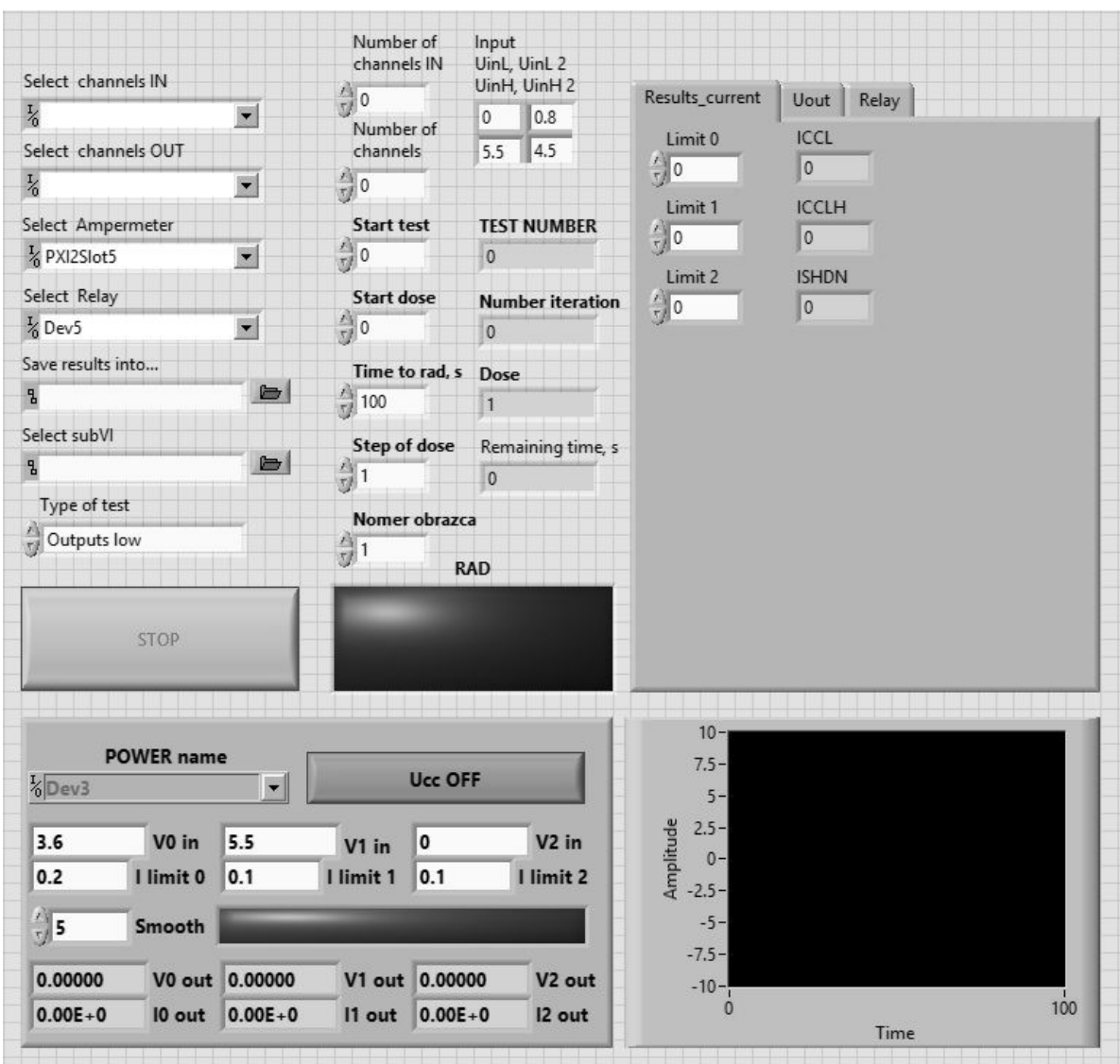

Figure 1. The control panel of the developed system. 
The specialized software contains several independent subprograms operating in parallel and communicating through the local variables:

- power source control subprogram sets the output voltage and maximum output current and performs continuous measurement of the actual values of output voltages and currents on the 3 channels, automatically switching the current measurement ranges on 2 channels and quickly disconnecting the power circuits in the case of latchup effect.

- NI-5105 - allows to monitoring the functioning of the chip charts in real time.

- Subprogram of the current measurements can automatically switch between all available current ranges of DMM PXI-4071 and carry out the current measurements of less than $1 \mathrm{nA}$ at a frequency down to $5 \mathrm{~Hz}$ (with down to 500 measurements in each point).

- The switch module subprogram ensures the correct relay switching and protects against switching collision.

- Subprogram of test algorithm determines the sequence of the test procedures, the sequence of parametric control, as well as the time of start / stop of the source of influence.

- NI-6723, NI-6229 and NI-6556 - the digital and analog data processing subprogram allows setting / reading of analog and digital signals.

- DUT mode, actual values and data timing are managed by the main algorithm subprogram.

- Subprogram of source of exposure control allows to initiate or stop the impact on chip. View subprogram determined by the type of exposure source.

\section{The typical results of tests performed by the developed system}

The tests results of the two IC studies, using the developed system, are listed below. The DUTs were SN74AHC14D and SN74LVC14ADR - 6 independent Schmitt-Trigger Inverters.

The AHC series (Advanced High-Speed CMOS - an advanced high speed CMOS structure) improves performance in low-speed input signal power in the presence of significant interference; provide additional protection against static discharge circuit and overload outlets and have a relatively large range of supply voltage [5].

The LVD series (Low Voltage CMOS) is specifically designed to work with lowvoltage and low power consumption [6]. The CMOS LVC family provides devices with supply voltage in the range from $3.3 \mathrm{~V}$ to $5 \mathrm{~V}$.

The radiation test was performed by using the Cs137 isotopic source "GammaPanorama MEPhI". During the experiment the parameters are contain in Table 1 were controlled.

The truth table for DUT is usually described in its datasheet.

It was found that the critical functional mode of both ICs is the regime providing low output static voltage. TID dependences of the supply current of both of the tested ICs are shown in Figure 2. The change of the other monitoring parameters with TID was insignificant. 


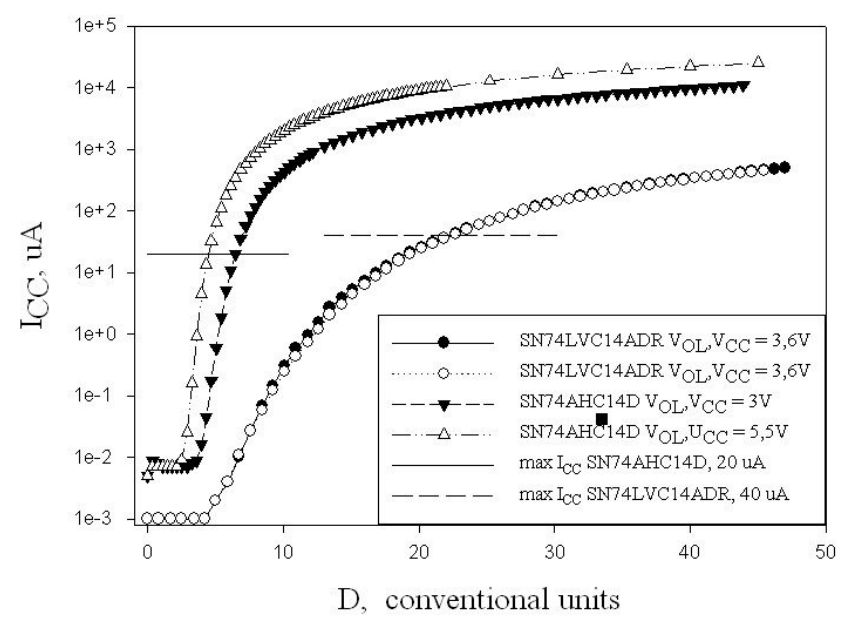

Figure 2. TID dependence of the supply current for tested ICs in various regimes.

The levels of parametrical failure have been determined for tested ICs. LVC devices possess more stable radiation behavior in comparison with AHC devices. Probably, such difference may be caused by presence of guard output structure in AHC series; that structures may be not optimized for TID influence.

Research in a range of temperatures was conducted using the developed system. The object of research was the logical IC - 6 logic elements "NOT" made by bipolar technology. Studies were carried out at ambient temperatures of $25^{\circ} \mathrm{C} \pm 10^{\circ} \mathrm{C}, 125^{\circ} \mathrm{C}$ and $-60^{\circ} \mathrm{C}$.

During operation it was found that the chips have a high radiation resistance. Figure 3 shows TID dependence of the supply current increment for tested ICs in various temperatures.

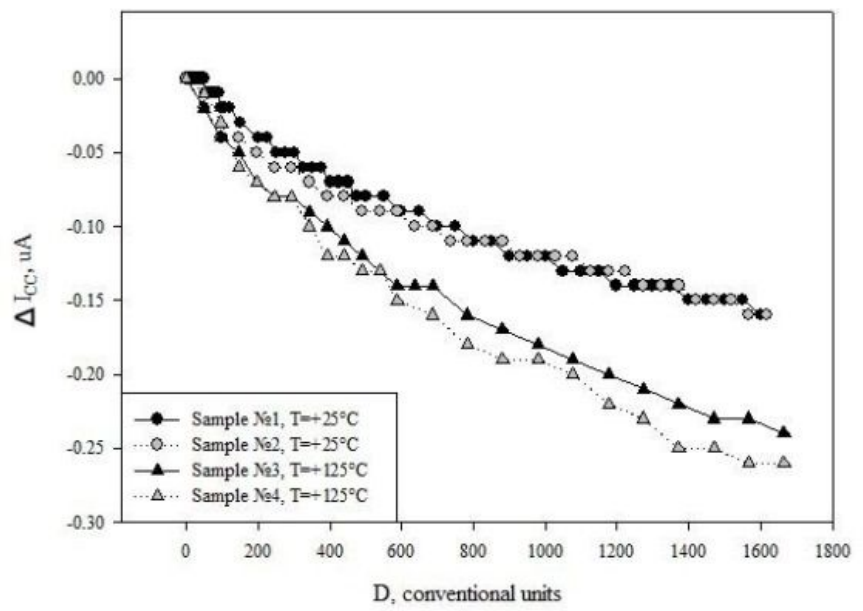

Figure 3. TID dependence of the supply current increment for tested ICs in various temperatures.

\section{Conclusions}

PXI platform equipment with LabVIEW development system is an optimal solution for engineering hardware and software control systems like described in the article. 
The developed system allows to carry out research at other environmental conditions and facilities (pulsed electron accelerators, electron LINACs, X-ray testers etc.)

The developed automated system has successfully implemented at JSC "ENPO SPELS", Moscow, Russia, and was used to test more than 20 IC types. The plan for further work in this direction is to expand the developed system on other IC types and to implement the elements of this system into existing systems for parametric control of electronic devices $[7,8]$.

\section{Acknowledgements}

The paper is a part of the research done within the government assignment given by the ministry of education and science of Russian Federation (ref. number RFMEFI57414X0115).

\section{References}

[1] A.Y. Nikiforov, D.V. Boychenko, V.A. Telets, Book of abstracts RAD-2014, 300 (2014)

[2] D.V. Boychenko, O.A. Kalashnikov, A.Y. Nikiforov, A.V. Ulanova, D.V. Bobrovsky, P.V. Nekrasov, Facta Universitatis Series: Electronics and Energetics 28 , $153(2015)$

[3] PXI Platform, National Instruments Corporation URL: http://sine.ni.com/np/app/main/p/ap/global/lang/ru/pg/1/sn/n24:PXI-FSLASHCompactPCI/

[4] G. G. Davydov, A. S. Kolosova, L. N. Kessarinskiy, D.V. Boychenko, 2015 International Siberian Conference on Control and Communications (SIBCON), 7147094 (2015) doi : 10.1109/SIBCON.2015.7147094

[5] AHC/AHCT Designer's Guide, TI, 2000 URL: http://www.ti.com/lit/ml/scla013d/scla013d.pdf.

[6] LVC Designer's Guide, TI, 1996 URL: http://www.ti.com/lit/ml/scba010/scba010.pdf.

[7] M. Z. Malik, A. Farooq, A. Ali, G. Chen, MATEC Web of Conferences 40, 07001 (2016) doi : 10.1051/matecconf/20164007001

[8] W. Song, W. Zhang, MATEC Web of Conferences 22, 03001 doi : $10.1051 /$ matecconf $/ 20152203001$ 